La revue La revue pour l'histoire du CNRS

POUR L'HISTOIRE DU CNRS $15 \mid 2006$

CNRS et Université

Sakharov Science morale et politique

Charles Rhéaume, Les Presses de l'Université Laval, 2004

Catherine Vilkas

\title{
CpenEdition
}

Journals

Édition électronique

URL : https://journals.openedition.org/histoire-cnrs/526

DOI : $10.4000 /$ histoire-cnrs.526

ISSN : 1955-2408

Éditeur

CNRS Éditions

Édition imprimée

Date de publication : 3 novembre 2006

ISBN : 978-2-271-06452-3

ISSN : $1298-9800$

Référence électronique

Catherine Vilkas, "Sakharov Science morale et politique », La revue pour I'histoire du CNRS [En ligne],

15 | 2006, mis en ligne le 22 novembre 2006, consulté le 20 mai 2021. URL : http://

journals.openedition.org/histoire-cnrs/526; DOI : https://doi.org/10.4000/histoire-cnrs.526

Ce document a été généré automatiquement le 20 mai 2021.

Comité pour I'histoire du CNRS 


\section{Sakharov Science morale et politique}

Charles Rhéaume, Les Presses de l'Université Laval, 2004

\section{Catherine Vilkas}

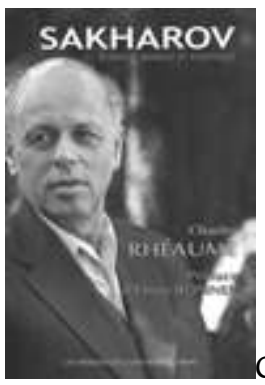

Comment un brillant physicien soviétique, choyé par le régime pour son rôle clé dans l'élaboration de la bombe à hydrogène de son pays, est-il devenu un symbole de la cause des droits de l'homme ? Face aux sanctions que sa lutte valut à Andreï Sakharov, Prix Nobel de la paix 1975, quelles furent les attitudes des scientifiques occidentaux, notamment américains, français et britanniques ? À partir de trente-sept entretiens et de nombreuses sources documentaires, Charles Rhéaume, lauréat 2005 du Prix Jean-Finot de l'Académie des sciences morales et politiques, répond de façon détaillée à ces questions. Il fait de Sakharov l'emblème du «scientifique de conscience » qui réussit à dépasser son endoctrinement idéologique et sacrifia sa position privilégiée au cœur de la technoscience soviétique au nom des principes universels de liberté et de justice.

Nous découvrons dans la première partie de l'ouvrage les traits de personnalité et le mode de pensée d'un homme dont l'originalité caractérise non seulement ses travaux en physique des particules mais aussi son activité sociale, qu'il veut toujours moralement justifiée et soumise à obligation de résultat. C'est ainsi que Sakharov échappe au sentiment de culpabilité, la bombe $\mathrm{H}$ ayant assuré l'équilibre entre l'Union soviétique et les États-Unis dans un objectif de paix mondiale. À ses qualités scientifiques rares s'ajoute l'héritage d'une intelligentsia russe favorable à la modernité occidentale issue des Lumières - Sakharov se perçoit d'ailleurs « libre penseur » plus 
que « dissident ». Sans omettre l'influence des milieux familial et d'études, puis le rôle de sa femme Elena Bonner à ses côtés, l'auteur le qualifie d'hybride réconciliant les cultures scientifique et littéraire, technocratique et humaniste.

2 Le lecteur partage dans la deuxième partie les doutes qui assaillent Sakharov en raison des essais nucléaires, du lyssenkisme et du retard de la science soviétique (hors des domaines liés à la technologie militaire) ; puis défilent les années du dégel, clôturées par l'invasion de la Tchécoslovaquie en 1968 alors que circulent les « Réflexions sur le progrès, la coexistence pacifique et la liberté intellectuelle ». Si les traces de rhétorique soviétique n'échappent pas à Charles Rhéaume, celui-ci y trouve aussi l'influence de l'ethos scientifique sur la vision politique de Sakharov, empreinte d'internationalisme et d'humanisme. La réception souvent enthousiaste du Manifeste par les scientifiques occidentaux n'apporte toutefois pas de réponses collectives à leur collègue de l'autre côté du rideau de fer. Celles-ci émergeront en 1973 face à la violente campagne de diffamation menée contre Sakharov, qui a entretemps aggravé considérablement son cas par ses appels à la démocratisation. L'auteur montre alors comment les échanges scientifiques internationaux, dont le gouvernement soviétique est friand, peuvent devenir un levier au service des dissidents : il s'agit de subordonner la coopération au respect des libertés scientifiques, étroitement articulées avec le droit humanitaire international que viennent enrichir les accords d'Helsinki de 1975.

3 L'ouvrage évite le piège d'une focalisation excessive sur le seul cas Sakharov lorsqu'il étudie les formes de soutien en France, en Grande- Bretagne et aux États- Unis. Très instructifs quant aux différentes attitudes des individus, des groupes et des institutions scientifiques, ces trois chapitres, tout comme la dernière partie consacrée à la période de l'exil intérieur de Sakharov, montrent bien les dilemmes que suscite la solution de la non coopération au sein d'une profession socialisée aux collaborations internationales. C'est alors la détermination des comités ad hoc qui s'avère efficace.

4 Seul bémol, le lecteur qui aurait souhaité approfondir le contenu et les conditions d'exercice de l'activité scientifique de Sakharov au cours de sa trajectoire serait resté sur sa faim. Mais tel n'était pas l'objectif de l'ouvrage, rédigé sous l'angle d'une " histoire morale de la science " qui ne manquera pas d'intéresser aussi bien ceux qui s'interrogent sur la responsabilité du scientifique que les historiens de la guerre froide et de la dissidence. 